\title{
Type 2 diabetes mellitus does not increase the risk of lung cancer among never-smokers: a nationwide cohort study
}

\author{
Hyung Jun Park ${ }^{1,2,3}$, Hee-Kyung Joh ${ }^{2,4,5}$, Seulggie Choi ${ }^{5}$, Sang Min Park ${ }^{5,6}$ \\ ${ }^{1}$ Department of Family Medicine, SMG - SNU Boramae Medical Center, Seoul, Republic of Korea; ${ }^{2}$ Department of Family Medicine, Seoul \\ National University Health Service Center, Seoul, Republic of Korea; ${ }^{3}$ Clinical Medical Sciences, College of Medicine, Seoul National University, \\ Seoul, Republic of Korea; ${ }^{4}$ Department of Medicine, Seoul National University College of Medicine, Seoul, Republic of Korea; ${ }^{5}$ Department of \\ Family Medicine, Seoul National University Hospital, Seoul, Republic of Korea; ${ }^{6}$ Department of Biomedical Science \& Family Medicine, Seoul \\ National University College of Medicine, Seoul, Republic of Korea \\ Correspondence to: Sang Min Park, MD, PhD. Department of Family Medicine, Seoul National University College of Medicine; Health Promotion \\ Center, Seoul National University Hospital; Department of Biomedical Science \& Family Medicine, Seoul National University College of Medicine, \\ 101 Daehangno, Jongno-gu, Seoul 110-744, Republic of Korea. Email: smpark.snuh@gmail.com.
}

\begin{abstract}
Previous studies have reported higher incidences of lung cancer among smokers diagnosed with diabetes than those without. Accordingly, this study investigated whether type 2 diabetes mellitus (T2DM) diagnosis alone can be associated with increased lung cancer incidences among never-smokers in the Korean population. Newly diagnosed never-smoking T2DM patients were identified from the nationwide longitudinal cohort of health examination data of South Korea (2002-2013). Cox proportional hazards regression model was employed to estimate the hazard ratios (HRs) and 95\% confidence interval (CI) of lung cancer in the T2DM patient and abnormal fasting blood sugar (FBS) groups. T2DM (HR =0.91, 95\% CI: 0.71 to 1.17) and abnormal FBS level are no significant association with lung cancer incidence based on the given HR. T2DM does not increase the risk of lung cancer among never-smokers. A large cohort study affirms minimal impact of T2DM on lung cancer development in the never-smoking Korean population.
\end{abstract}

Keywords: Lung cancer; type 2 diabetes mellitus cohort analysis (T2DM cohort analysis); longitudinal study

Submitted May 16, 2019. Accepted for publication Oct 12, 2019.

doi: $10.21037 /$ tlcr.2019.11.01

View this article at: http://dx.doi.org/10.21037/tlcr.2019.11.01

\section{Introduction}

Lung cancer is the most frequently diagnosed cancer and the leading cause of cancer-related death among both male and female populations worldwide (1). Smoking is well known as the major risk factor for lung cancer and other factors such as insulin resistance and type 2 diabetes have reported to be increase lung cancer risk in smokers (2).

Among smoking-unrelated risk factors for lung cancer development, diabetes mellitus is considered an independent risk factor (3). However, contradictory and often conflicting findings have been reported in cohort studies (4).

The Korean National Health Insurance Services (NHIS), as a single-payer health insurance system, covers almost the entire population of South Korea. NHIS also provides biennial health examinations to any individuals aged 40 years and older. Medication exposure of an individual can also be obtained from the NHIS claims database which contains prescription records.

Using this database, this study aimed to evaluate the effect of type 2 diabetes mellitus (T2DM) and abnormal fasting blood sugar (FBS) level on lung cancer incidences within the never-smoker Korean papulations.

\section{Methods}

We analyzed NHIS data from a standardized a 12-year longitudinal cohort of health examination to investigate the effects of T2DM and abnormal FBS levels on lung cancer among the nonsmoker population. Figure 1 shows 


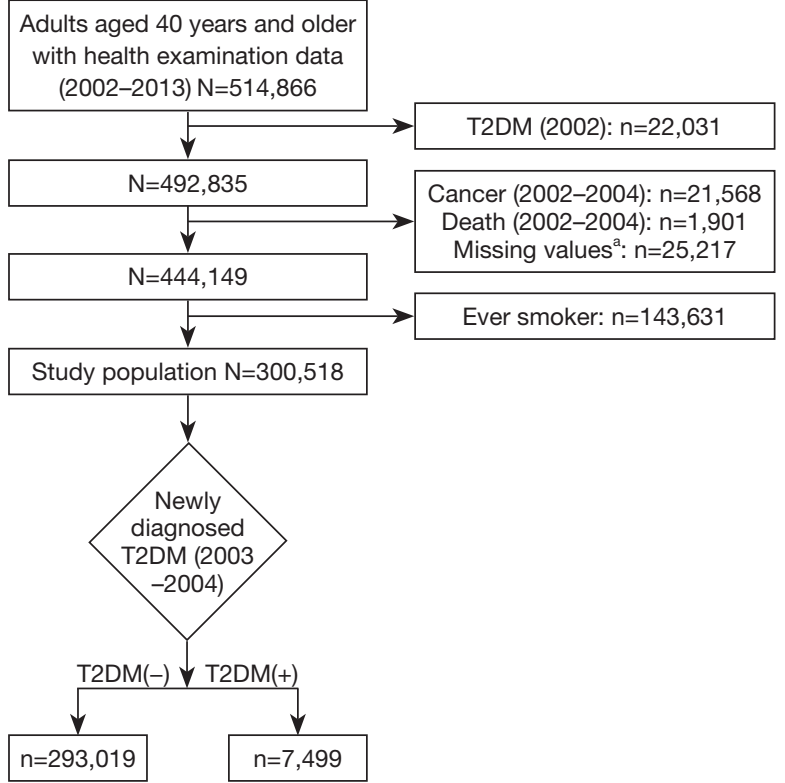

Figure 1 Flow chart of inclusion in the study population. a, missing values for sociodemographic characteristics (age, gender, household income), behavioral factors (alcohol consumption, tobacco smoking, physical activity), and medical conditions (CCI score, body mass index, type 2 diabetes, systolic blood pressure, FBS, total serum cholesterol). T2DM, type 2 diabetes mellitus; FBS, fasting blood sugar; CCI, Charlson Comorbidity Index.

a schematic flow chart of data extraction and inclusion criteria.

T2DM patient population was identified by anti-diabetic medication prescription, as outlined by the International Classification of Diseases code-10th Revision (ICD-10) pertaining to T2DM (E11-E14). Data from the first year (January 1, 2002 to December 31, 2002) was used to washout diagnosed T2DM patients in order to exclude those who already had T2DM for an unknown period prior to the start date. Any cancer patients and participants who passed away during the first three years (January 1, 2002 to December 31, 2004) were excluded to minimize bias on incidence and death of lung cancer. The never-smoker population was defined as individuals who self-reported to have no experience in smoking. As such, we excluded the ever-smoker populations. T2DM patients defined as those who were newly diagnosed T2DM at the period from January 1, 2003 to December 31, 2004. In addition, the association between fasting blood glucose and lung cancer was also analyzed because of some participants that were not diagnosed with T2DM, but showed abnormal FBS levels.
The cohort entry date was defined as January 1, 2005, and the follow-up observation period was extended to December 31, 2013. Throughout this period, newly diagnosed lung cancer patients were identified to avoid immortal time bias. Lung cancer patients were defined as individuals who visited outpatient $\geq 2$ times, or were hospitalized at least once or passed away with a C34 ICD10 code. Sociodemographic, medical, and health-related behavior data from 2003 to 2004 (before cohort entry date) were extracted.

Cox proportional hazards regression analysis with T2DM status and FBS status as exposures were employed to estimate hazard ratios (HRs) and $95 \%$ confidence interval (CI). FBS status was defined as hypoglycemic $(<60 \mathrm{mg} / \mathrm{dL})$, normal $(\geq 60,<100 \mathrm{mg} / \mathrm{dL})$, prediabetic $(\geq 100,<125 \mathrm{mg} / \mathrm{dL})$, and diabetic level $(\geq 126 \mathrm{mg} / \mathrm{dL})$.

Statistical analyses were performed using STATA software version 14.0 (Stata Co., College Station, TX, USA), and $\mathrm{P}$ values less than 0.05 were considered statistically significant.

\section{Results}

Table 1 shows the baseline characteristics of study population separated by T2DM status within the never-smoker population. A total of 300,518 participants (103,288 men and 197,230 women) included in our analysis.

Unadjusted model shows T2DM associated with incidence of lung cancer based on HRs (Table 2). FBS status at the level of prediabetes and diabetes are associated with lung cancer regardless of diagnosis of T2DM. On the other hands, hypoglycemic levels of FBS are not associated with lung cancer development. Interestingly, FBS levels of prediabetes and diabetes of undiagnosed T2DM group have higher HRs than those of diagnosed T2DM group in the unadjusted model.

However, the multivariable model shows that T2DM is not significantly associated with the incident of lung cancer based on HRs (HR $=0.91,95 \%$ CI: 0.71 to 1.17 ; Table 2). Furthermore, abnormal FBS status is also not significantly associated with lung cancer, regardless of participants' diagnosis of T2DM (Table 2).

\section{Discussion}

The participants who were not diagnosed with T2DM between 2003 and 2004, but whose fasting blood glucose had reached diabetic levels at the baseline year estimated 
Table 1 Baseline characteristics of study population according to type 2 diabetes status in never-smokers

\begin{tabular}{|c|c|c|c|}
\hline Characteristics & No type 2 diabetes & Type 2 diabetes & $P$ value \\
\hline Number & 293,019 & 7,499 & \\
\hline \multicolumn{4}{|l|}{ Sex } \\
\hline Male & $100,112(34.2)$ & 3,176 (42.4) & $<0.001$ \\
\hline Female & $192,907(65.8)$ & 4,323 (57.7) & \\
\hline \multicolumn{4}{|l|}{ Age (year) } \\
\hline $40-49$ & $111,547(38.1)$ & 1,217 (16.2) & $<0.001$ \\
\hline $50-59$ & 91,264 (31.1) & 2,348 (31.3) & \\
\hline$\geq 60$ & 90,208 (30.8) & $3,934(52.5)$ & \\
\hline \multicolumn{4}{|c|}{ Household income ${ }^{a}$} \\
\hline $1 \mathrm{Q}$ (the highest) & 97,501 (33.3) & 2,291 (30.6) & $<0.001$ \\
\hline $2 Q$ & $83,251(28.4)$ & $2,266(30.2)$ & \\
\hline $3 Q$ & 64,087 (21.9) & $1,604(21.4)$ & \\
\hline 4Q (the lowest) & $48,180(16.4)$ & 1,338 (17.8) & \\
\hline \multicolumn{4}{|l|}{ Alcohol } \\
\hline Never drinker & 211,086 (72.0) & $5,534(73.8)$ & $<0.001$ \\
\hline Ever drinker & 81,933 (28.0) & 1,965 (26.2) & \\
\hline \multicolumn{4}{|l|}{ Exercise $^{b}$} \\
\hline Low & $173,844(59.3)$ & $4,379(58.4)$ & $<0.001$ \\
\hline Moderate & $89,951(30.7)$ & $2,069(27.6)$ & \\
\hline High & $29,224(10.0)$ & $1,051(14.0)$ & \\
\hline $\begin{array}{l}\text { Charlson } \\
\text { Comorbidity } \\
\text { Index }^{c}\end{array}$ & $0.69 \pm 0.90$ & $1.57 \pm 1.29$ & $<0.001$ \\
\hline \multicolumn{4}{|l|}{$\mathrm{BMI}^{\mathrm{d}}, \mathrm{kg} / \mathrm{m}^{2}$} \\
\hline$<18$ & 3,750 (1.3) & $50(0.7)$ & $<0.001$ \\
\hline$\geq 18,<23$ & $108,496(37.0)$ & $1,780(23.7)$ & \\
\hline$\geq 23<25$ & 79,945 (27.3) & 1,953 (26.0) & \\
\hline$\geq 25$ & $100,828(34.4)$ & 3,716 (49.6) & \\
\hline
\end{tabular}

Blood pressure, $\mathrm{mmHg}$

Systole

$\begin{array}{llll}<140 & 218,891(74.7) & 4,582(61.1) & <0.001 \\ \geq 140 & 74,128(25.3) & 2,917(38.9)\end{array}$

Total cholesterol, $\mathrm{mg} / \mathrm{dL}$

$\begin{array}{lrrr}<240 & 251,712(85.9) & 5,946(79.3) & <0.001 \\ \geq 240 & 41,307(14.1) & 1,553(20.7) & \end{array}$

Table 1 (continued)
Table 1 (continued)

\begin{tabular}{lccc}
\hline Characteristics & No type 2 diabetes & Type 2 diabetes & $P$ value \\
\hline \multicolumn{4}{l}{ Fasting blood sugar, $\mathrm{mg} / \mathrm{dL}$} \\
$<60$ & $482(0.2)$ & $2(0.0)$ & $<0.001$ \\
$\geq 60,<100$ & $214,110(73.1)$ & $1,418(18.9)$ & \\
$\geq 100,<125$ & $66,859(22.8)$ & $2,072(27.6)$ & \\
$\geq 126$ & $11,037(3.8)$ & $3,955(52.7)$ & \\
Lung cancer & & & \\
$(-)$ & $291,113(99.3)$ & $7,433(99.1)$ & 0.015 \\
$(+)$ & $1,906(0.7)$ & $66(0.9)$ & \\
\hline
\end{tabular}

Data are number (\%) or mean \pm standard deviation. ${ }^{a}$, income quartiles based on the imposed of national health insurance premiums on each household; ' , exercise status according to the frequency of weekly exercise (low: 0 times, moderate: 1-4 times, high: 5-7 times); ' , calculated from acute myocardial infarction, congestive heart failure, peripheral vascular disease, cerebral vascular accident, dementia, pulmonary disease, connective tissue disorder, peptic ulcer disease, liver disease, diabetes mellitus, diabetes complications, hemiplegia, renal disease, severe liver disease, and HIV based on ICD-10 codes of hospital visits from 2002 to 2004 ; ${ }^{d}$, body mass index cut-offs specific to the South Korean population ( $<18$ : underweight; $\geq 18$, $<23$ : normal; $\geq 23,<25$ : overweight; $\geq 25$ : obese).

that participants might not have kept their fasting time well at the time of the blood test or missed proper diagnosis of diabetes after the test. Therefore, in order to compensate for such ambiguity, we also analyzed the effects of FBS on lung cancer regardless of the diagnosis of T2DM.

Unadjusted analysis of T2DM and FBS level of prediabetes and diabetes on lung cancer development shows significant association. However, all results were statistically insignificant after adjusting for age, gender and other covariables. This suggests that the incidence of lung cancer in nonsmokers can be driven by age regardless of diabetes or blood sugar level.

A never-smoker commonly defined as participants who smoked less than 100 cigarettes over the course of their lifetime by the previous study (5). However, as smoking status data was given by self-reporting questionnaire, we excluded ex-smokers to classify the never-smoker to minimize recall bias in this study.

In this study, data on tumor stage and pathology of tumors could not be obtained, which could not be analyzed. However, since the results of this study included newly 
Table 2 Hazard ratios for lung cancer according to type 2 diabetes and fasting blood sugar status

\begin{tabular}{|c|c|c|c|}
\hline Type 2 diabetes and fasting blood sugar status & \multicolumn{3}{|c|}{ Hazard ratio (95\% confidence interval) } \\
\hline Type 2 diabetes & $1.40(1.09-1.79)$ & $0.94(0.73-1.20)$ & $0.91(0.71-1.17)$ \\
\hline \multicolumn{4}{|l|}{ FBS status of participants diagnosed with T2DM } \\
\hline Hypoglycemic (<60 mg/dL) & $1.06(0.51-2.23)$ & $0.81(0.39-1.71)$ & $0.76(0.36-1.59)$ \\
\hline Prediabetic ( $\geq 100,<125 \mathrm{mg} / \mathrm{dL})$ & $1.16(1.09-1.24)$ & $0.97(0.91-1.04)$ & $1.01(0.95-1.09)$ \\
\hline Diabetic ( $\geq 126$ mg/dL) & $1.47(1.32-1.65)$ & $1.03(0.92-1.15)$ & $1.05(0.94-1.18)$ \\
\hline \multicolumn{4}{|l|}{ FBS status of participants not diagnosed withT2DM } \\
\hline Hypoglycemic (<60 mg/dL) & $1.72(0.71-4.14)$ & $1.45(0.60-3.48)$ & $1.40(0.58-3.38)$ \\
\hline Diabetic ( $\geq 126$ mg/dL) & $1.60(1.31-1.95)$ & $1.06(0.87-1.29)$ & $1.09(0.89-1.33)$ \\
\hline
\end{tabular}

${ }^{a}$, adjusted for sociodemographic characteristics (age, gender, household income), behavioral factors (alcohol consumption, physical activity), and medical conditions (CCI score, body mass index, type 2 diabetes, systolic blood pressure, total serum cholesterol). T2DM, type 2 diabetes mellitus; FBS, fasting blood sugar; $\mathrm{CCl}$, Charlson Comorbidity Index.

diagnosed lung cancer and deaths from lung cancer, the association of T2DM and lung cancer development could be found regardless of tumor stage.

Previous studies have shown the contradictory effects depending on diabetes medication on the incidence of lung cancer $(6,7)$ Although, we did not analysis the effect of each anti-diabetic medication on the incidence of lung cancer, the impact of diabetic FBS levels on lung cancer development was consistently statistically insignificant, regardless of regardless of anti-diabetic medication prescription among never-smokers, based on the multivariable adjusted HR.

A previous review study has identified several factors associated with lung cancer incidence within never-smokers such as: environmental tobacco smoke, occupational exposure to the carcinogen, radon exposure, indoor or outdoor air pollution, as well as genetic factors (5). This study did not take into account the above factors associated with lung cancer in never-smokers, and thus could pose as potential limitations. However, the merits of this study lie in the adjustment of considerable factors such as medical conditions, sociodemographic and behavioral factors within the large-scale longitudinal national cohort data pool. Furthermore, given that the previous study that lung cancer incidence in never-smokers is associated within the Caucasian population (8), this study suggests that lung cancer development in never-smokers in the Korean population is not associated with T2DM or FBS levels.

\section{Acknowledgments}

Funding: This study used data from the Korean National Health Insurance Service (NHIS-2017-2-459). The protocol employed in the present study was approved by the Institutional Review Board of Seoul National University Hospital (IRB number: 1703-039-836).

\section{Footnote}

Conflicts of Interest: The authors have no conflicts of interest to declare.

Ethical Statement: The authors are accountable for all aspects of the work in ensuring that questions related to the accuracy or integrity of any part of the work are appropriately investigated and resolved.

\section{References}

1. Bray F, Ferlay J, Soerjomataram I, et al. Global cancer statistics 2018: GLOBOCAN estimates of incidence and mortality worldwide for 36 cancers in 185 countries. CA Cancer J Clin 2018;68:394-424. 
2. Yu W, Wang Z, Qian L, et al. HRCT features distinguishing minimally invasive adenocarcinomas from invasive adenocarcinomas appearing as mixed ground-glass nodules. Transl Cancer Res 2018;7:1298-305.

3. Pallis AG, Syrigos KN. Lung cancer in never smokers: disease characteristics and risk factors. Crit Rev Oncol Hematol 2013;88:494-503.

4. Yang WS, Yang Y, Yang G, et al. Pre-existing type 2 diabetes and risk of lung cancer: a report from two prospective cohort studies of 133024 Chinese adults in

Cite this article as: Park HJ, Joh HK, Choi S, Park SM. Type 2 diabetes mellitus does not increase the risk of lung cancer among never-smokers: a nationwide cohort study. Transl Lung Cancer Res 2019;8(6):1073-1077. doi: 10.21037/tlcr.2019.11.01 urban Shanghai. BMJ Open 2014;4:e004875.

5. Subramanian J, Govindan R. Lung cancer in never smokers: a review. J Clin Oncol 2007;25:561-70.

6. Tseng C-H. Metformin and lung cancer risk in patients with type 2 diabetes mellitus. Oncotarget 2017;8:41132.

7. Ozerlat I. Diabetes therapy and lung cancer. Nature Reviews Endocrinology 2012;8:445.

8. Pirie K, Peto R, Green J, et al. Lung cancer in never smokers in the UK Million Women Study. Int J Cancer 2016;139:347-54. 